\title{
Effects of task and hip-abductor fatigue on lower limb alignment and muscle activation
}

\author{
Rodrigo Rabello ${ }^{1}$ (C) Camila Nodari ${ }^{2} \cdot$ Felipe Scudiero $^{2} \cdot$ Iury Borges $^{2} \cdot$ Luan Fitarelli $^{2} \cdot$ Jean Bianchesse ${ }^{2}$. \\ Rodrigo Rodrigues ${ }^{2,3}$
}

Received: 25 June 2021 / Accepted: 5 October 2021 / Published online: 15 October 2021

(c) The Author(s) 2021

\begin{abstract}
Purpose Fatigue-induced hip-abductor weakness may exacerbate lower-limb misalignments during different dynamic singleleg tasks. We sought to evaluate the effects of fatigue and task on lower limb kinematics and muscle activation and to find associations between measurements obtained in two tasks.

Methods One-group pretest-posttest design. Seventeen healthy adults $(9 \mathrm{~W})$ performed the single-leg squat $\left(\mathrm{SL}_{\mathrm{SQUAT}}\right)$ and the single-leg hop ( $\mathrm{SL}_{\mathrm{HOP}}$ ) before and after a hip-abduction fatigue protocol. Hip adduction, knee frontal plane projection angle (knee FPPA) and heel inversion displacement were measured during the eccentric phase of the $\mathrm{SL}_{\mathrm{SQUAT}}$ and the $\mathrm{SL}_{\mathrm{HOP}}$, as well as activation of the gluteus medius (GMed), tensor fascia latae (TFL), peroneus longus (PER) and tibialis anterior (TA). Moments and tasks were compared using a repeated-measures two-way ANOVA. Correlation between tasks was evaluated using Spearman's correlation.

Results No differences in kinematics or activation were found between moments. Hip-adduction displacement $(P=0.005)$, GMed $(P=0.008)$ and PER $(P=0.037)$ activation were higher during $\mathrm{SL}_{\mathrm{SQUAT}}$, while TA activation was higher during $\mathrm{SL}_{\mathrm{HOP}}$ $(P<0.001)$. No differences were found between tasks in knee FPPA and heel inversion. Hip-adduction and knee FPPA were not correlated between tasks, while ankle inversion displacement was positively correlated $\left(r_{\mathrm{s}}=0.524-0.746\right)$.

Conclusion Different characteristics of $\mathrm{SL}_{\mathrm{SQUAT}}$ (slower and deeper) seem to have led to increased hip adduction displacement, GMed, and PER activation and decreased TA activation, likely due to higher balance requirements. However, hipabductor fatigue didn't influence lower-limb alignment during the tasks. Finally, evaluations should be performed with different single-leg tasks since they don't give the same lower-limb alignment information.
\end{abstract}

Keywords Hip-abductor weakness $\cdot$ Lower limb alignment $\cdot$ Single-leg $\cdot$ Localized fatigue

\section{Introduction}

Dynamic single-leg weight-bearing tasks are commonly used in the literature to identify misalignments that have been considered risk factors for lower limb injuries [1-5]. It has been suggested that hip-abductor weakness is associated with increases in the dynamic knee valgus measured

Rodrigo Rabello

rabello.rrs@gmail.com

1 Department of Biomedical Sciences for Health, University of Milan, Via Luigi Mangiagalli, 31, Milan, MI, Italy

2 Exercise Physiology and Physical Assessment Laboratory, Serra Gaúcha University Center, Caxias do Sul, RS, Brazil

3 Integrated Colleges of Taquara (FACCAT), Taquara, RS, Brazil by the frontal plane projection angle (FPPA), likely due to the resulting greater hip adduction [6]. In turn, excessive dynamic knee valgus has been associated with patellofemoral pain [1] and higher risk of anterior cruciate ligament injury in athletes [7]. In addition, altered ankle biomechanics have been found in individuals with lower hip-abductor strength, due to a higher contribution required from this joint to maintain balance [8].

When in the single-leg stance, the trunk tends to tilt medially about the acetabulum, dislocating the center of mass $(\mathrm{CoM})$ outside the base of support. To counteract this tendency and avoid loss of balance, the hip-abductors contract, generating a force that repositions the CoM inside the base of support [9]. This role of stabilizing the pelvis and trunk to maintain balance is performed in large part by the hipabductors (approximately 66\%), which include the gluteus 
medius and the tensor fascia latae [10]. In addition, the peroneus longus and the tibialis anterior also play a role in maintaining balance, by acting on the ankle joint to fine-tune the CoM's position and preserve adequate foot contact to the supporting surface $[8,11]$.

Several studies have used fatigue protocols of the hipabductors to try to understand how decreased force production capacity of these muscles influence frontal plane kinematics during different landing tasks, however, the findings weren't consistent. Jacobs et al. [12] found increased hipadduction displacement but no differences in knee valgus during single-leg landing after fatigue. Meanwhile, Geiser et al. [3] also observed greater hip displacement after fatigue in different landing tasks, however, they also found greater knee abduction displacement in the same conditions. Similarly, another study reported greater knee abduction angle after fatigue at $60 \mathrm{~ms}$ after initial contact in a single-leg jumping task, finding no differences in hip adduction angle [5]. Additionally, Lee and Powers [13] reported increased center of pressure displacement in a single-leg landing task when the hip muscles were fatigued. The lack of agreement between studies is likely due to the different tasks performed, as well as to the different fatiguing protocols used, which ranged from maximal exercises performed in an isokinetic dynamometer to protocols performed for many repetitions with no extra load. It is important to note, though, that hip abductor fatigue is used only as a surrogate of muscle weakness and is not an exact replication. Although isolated fatigue protocols decrease force production capacity $[14,15]$, subjects with natural weakness can develop compensation mechanisms that may alter lower limb alignment [3]. However, fatigue-induced hip abductor weakness allows researchers to identify a cause-and-effect relationship between weakness and biomechanics, which is not possible when cross-sectionally evaluating subjects that already present hip-abductor weakness [5].

A high number of functional tasks have been adopted in the literature to assess lower limb alignment. The single-leg squat $\left(\mathrm{SL}_{\mathrm{SQUAT}}\right)$ is a very popular task due to its simplicity and strong correlation to knee valgus angles during running and cutting tasks [2]. However, because of the low velocities achieved during this test, the $\mathrm{SL}_{\mathrm{SQUAT}}$ may give different information regarding lower limb alignment than other single-leg tasks. The single-leg hop $\left(\mathrm{SL}_{\mathrm{HOP}}\right)$ test presents as a more dynamic alternative [16]. Although the jumps' landing phases are more often used in the literature (because of the higher loads involved), the propulsion phase also puts enough stress on the lower limbs to expose eventual misalignments, which is the ultimate goal of functional tasks [17]. Furthermore, the $\mathrm{SL}_{\mathrm{HOP}}$ propulsion phase is somewhat similar to the $\mathrm{SL}_{\mathrm{SQUAT}}$, making comparisons more viable.

Although single-leg tasks seem to be an important tool to evaluate lower limb misalignments in both pre- and post-fatigue situations, it is not clear how kinematic parameters change when performing similar tasks at different speeds. Therefore, the primary aim of this study was to compare lower limb alignment parameters (hip adduction, knee FPPA, and heel inversion angles), as well as the activation of four muscles acting on the joints of interest, between the $\mathrm{SL}_{\mathrm{SQUAT}}$ and the $\mathrm{SL}_{\mathrm{HOP}}$ and before and after a hip-abductor fatigue protocol. A secondary aim was to identify if the measurements obtained during the two tasks are correlated to each other, to understand if results obtained from one task can be extrapolated to the other.

\section{Methods}

\section{Participants}

The appropriate sample size was estimated a priori with the G*Power software (version 3.1.9.6; University of Trier, Trier, Germany). The ANOVA: Repeated measures, withinbetween interaction, set with a significance level of $P=0.05$, power $1-\beta=0.85$ and an effect size $f$ of 0.42 (according to the results from Geiser et al. [3] for a pre/post hip fatigue knee abduction displacement comparison) indicated a sample size of 16. One additional participant was recruited to account for possible data loss. Recruitment was performed through publications on social media, local gyms, and the university campus. Participants had no history of lower limb injuries and had been participating in strength training programs for at least 3 months at the moment of data collection. No participant reported executing the study's tasks in their routine training. The study followed the principles of the Declaration of Helsinki and, after having the procedures explained to them, all participants gave written consent to participate in the study, which was approved by the university's ethics committee (number 76759817.7.0000.5668).

\section{Procedures}

STROBE guidelines were followed to strengthen the reporting of this study (Supplementary material 1). The study presents a one-group pretest-posttest design in which all subjects attended the laboratory in 3 days, with an interval of at least 7 days between each meeting. All data were collected between the months of September and November of 2018. On the first day, participants underwent body composition assessment and completed a ten-repetition maximum test (10RM) in the side-lying hip-abduction exercise, which was the exercise chosen for the fatigue protocol. On day two, subjects were familiarized with the maximum voluntary isometric contractions (MVICs) and the two single-leg tasks. And on the third day, participants performed: (1) the MVICs to be used for normalization of the EMG signal, 
(2) the two pre-fatigue single-leg tests, (3) the hip-abductor fatigue protocol and (4) the post-fatigue single-leg tests. A flow chart of the protocol steps is presented in Fig. 1. All tasks and measurements were performed on the subject's dominant limb, which was defined with the question "which foot would you use to kick a ball as far as possible?".

\section{Load definition}

On the first day, the load to be used for the fatigue protocol was determined by performing the 10RM test of the sidelying hip abduction exercise. In the lateral decubitus position, subjects warmed-up and were familiarized with the movement speed. They were instructed to abduct the hip to the maximum of their range of movement at a 60 beats per minute tempo $(2$ beats $=$ concentric phase; 2 beats $=$ eccentric phase), which was controlled by a metronome. The 10RM was defined as the load with which subjects could perform 10 well-executed repetitions, reaching concentric fatigue in the 11 th repetition. An initial load was chosen based on the subjects' previous experience. According to the number of repetitions performed with the initial load, the one used for the next trial was determined, until the precise number of repetitions was reached. All loads were determined using a maximum of three trials, with a 5-min rest between them [18].

\section{Familiarization}

On the second day, participants were familiarized with the MVICs and the single-leg tasks. After having the single-leg squat and the single-leg hop explained, participants executed them until they felt comfortable in performing both tasks. Afterwards, they were asked to perform the future protocol two times, executing five repetitions of the single-leg squat and three valid repetitions of the single-leg hop with an interval of approximately $10 \mathrm{~min}$ between the two blocks.
Three MVICs trials were performed for each muscle prior to the single-leg tasks.

\section{EMG data acquisition}

A Miotool-400 electromyographer (Miotec-Biomedical Equipment, Porto Alegre, Brazil), with four channels, sampling rate of $2 \mathrm{kHz}$ and 14-bits resolution, was used. During MVICs and the single-leg tests, activation of the gluteus medius, tensor fascia latae, peroneus longus and tibialis anterior were measured using pairs (interelectrode distance $=2 \mathrm{~cm}$ ) of pre-amplified surface electrodes with a bipolar configuration (Kendall Mini Medi-Trace 100Tyco Healthcare, São Paulo, Brazil). Skin preparation and electrode positioning were performed following the recommendations from the Surface ElectroMyoGraphy for the Non-Invasive Assessment of Muscles (SENIAM) [19]. A reference electrode was also positioned at the tibial tuberosity.

\section{Maximal voluntary isometric contractions}

Three MVICs were performed for each of the four muscles of interest. Gluteus medius and tensor fascia latae maximal activations were determined with isometric hip-abduction in the lateral decubitus position (Fig. 2A). Peroneus longus maximal activation was measured during isometric ankle eversion in the seated position (Fig. 2B) and tibialis anterior was determined with an isometric dorsiflexion while standing up (Fig. 2C). All MVICs were performed for $5 \mathrm{~s}$ while verbal encouragement was given, with a 2-min interval being observed after each contraction. For each muscle, the highest recorded EMG value obtained in the three trials was considered the maximal activation and used for normalization of the EMG data during the single-leg tests. Isometric hip-abduction was also performed after the fatigue protocol to verify its effects on the gluteus medius and tensor fascia latae muscles.
Fig. 1 Flow chart of the protocol steps

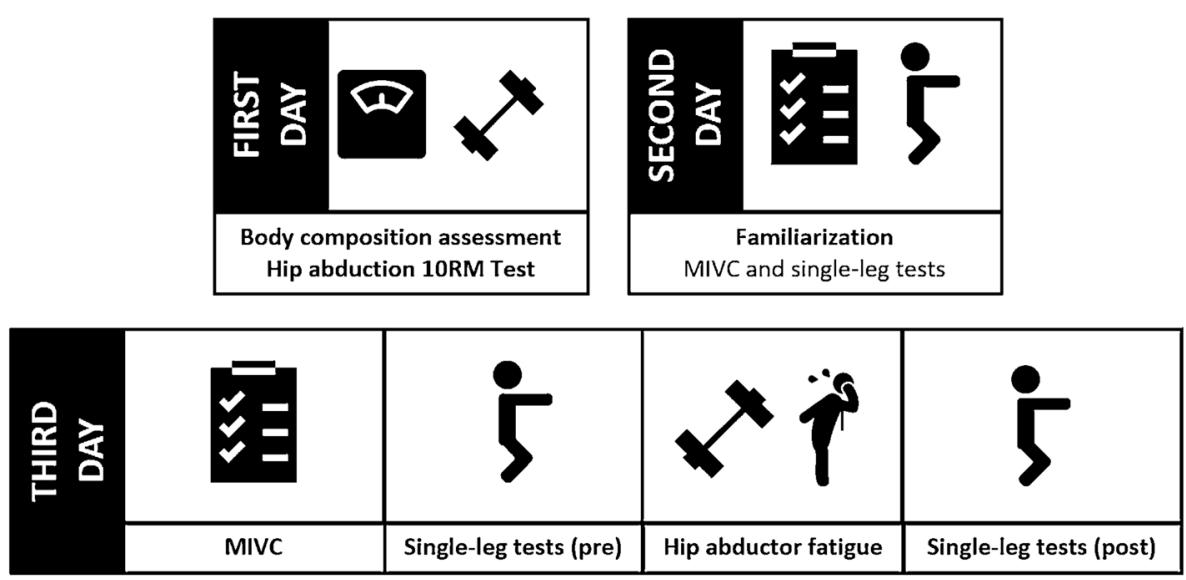



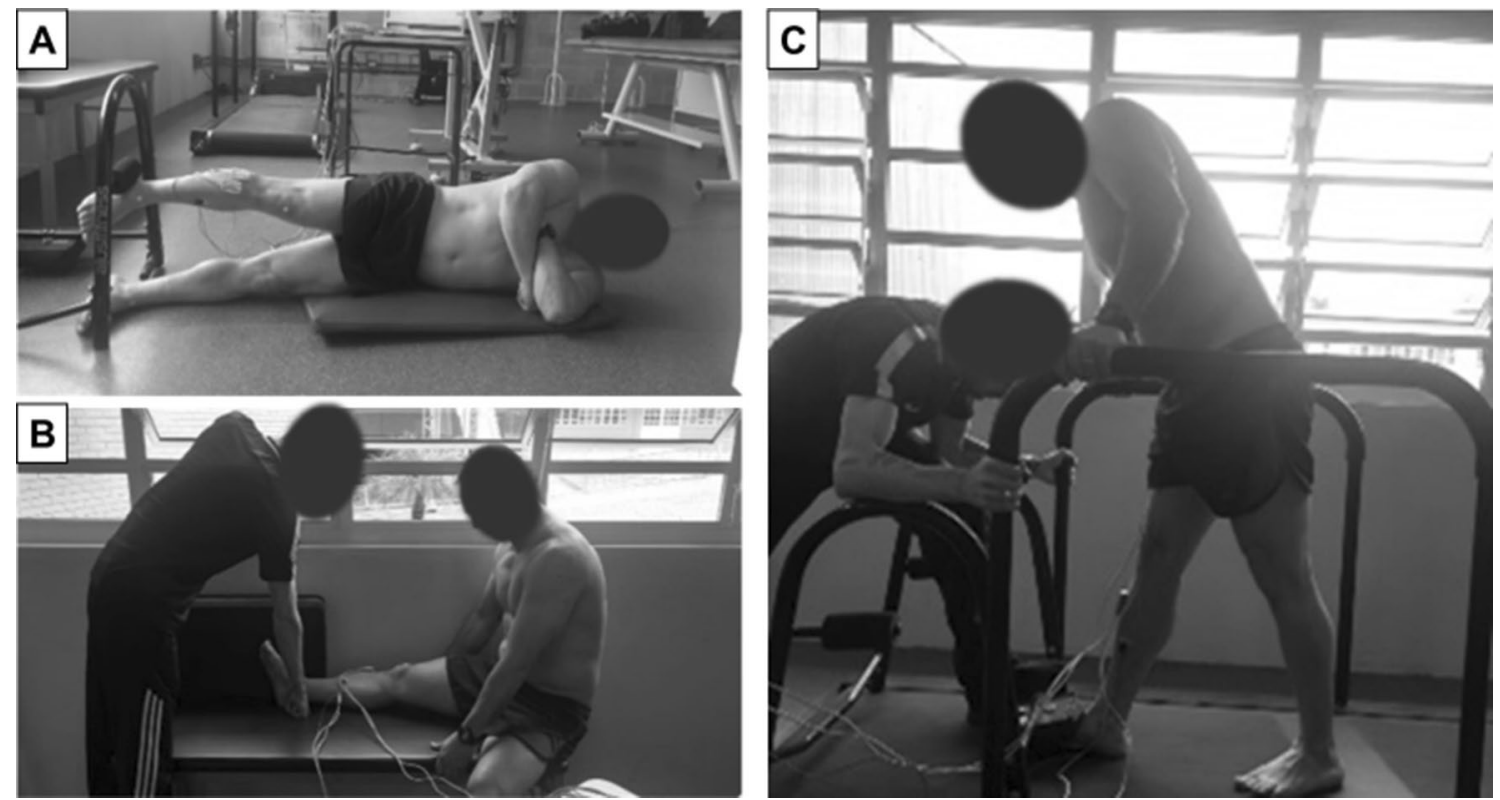

Fig. 2 Isometric contractions used for EMG normalization for the gluteus medius and the tensor fascia latae (A), the peroneus longus (B) and the tibialis anterior $(\mathbf{C})$

\section{Kinematic analysis of the single-leg tasks}

Two-dimensional kinematic analysis was used to evaluate the angles during the two single-leg tasks: a metronomecontrolled squat $\left(\mathrm{SL}_{\mathrm{SQUAT}}\right)$ and the propulsion phase of a single-leg hop ( $\left.\mathrm{SL}_{\mathrm{HOP}}\right)$. To measure hip adduction angle and knee FPPA, non-reflective markers were positioned on the anterior superior iliac spines (ASISs), on the center of the patella and at the midpoint of the ankle malleoli [20]. To evaluate heel inversion angle, a modified version of the protocol proposed by Perry \& Lafortune [21] was employed. Markers were positioned (1) $20 \mathrm{~mm}$ from the ground in the central region of the calcaneus, (2) $50 \mathrm{~mm}$ from the ground in the central region of the calcaneus, (3) in the Achilles tendon at lateral malleolus height and (4) in the center of the leg, $150 \mathrm{~mm}$ above the previous mentioned marker.

When performing the $\mathrm{SL}_{\mathrm{SQUAT}}$, participants were instructed to keep both feet on the ground until the researcher's command. When the command was given, they shifted their weight to the dominant side and flexed the contralateral knee, while keeping the trunk in a vertical position [20]. Participants then performed five, maximal depth, single-leg squats in sequence. Speed was controlled using a metronome ( 2 beats $=$ eccentric phase; 2 beats $=$ concentric phase $)$ and participants were allowed to move their arms as desired during the movement.

For $\mathrm{SL}_{\mathrm{HOP}}$ execution, the starting instructions and arm position were the same. However, in this case, participants were instructed to perform a single-leg hop as far as possible and to land without losing balance [16]. Three valid hops were performed with no rest between trials.

The order in which the tasks were performed was randomized for all participants by choosing one of two pieces of paper. For every participant, the order chosen for the pre-fatigue moment was repeated for the post-fatigue moment. The post-fatigue tasks were performed no more than two minutes after the post-fatigue hip-abduction MVICs, which, in turn, were performed no more than one minute after the end of the last set of hip-abductor fatigue exercise.

Three cameras recorded the execution of the trials to measure hip, knee and heel angles and to synchronize the phases of the movement with the EMG signal. One camera (JVC EverioS GZ-MS110; $30 \mathrm{~Hz}$ ) was positioned in the frontal plane, $3 \mathrm{~m}$ away at a $1 \mathrm{~m}$-height, with an anterior view of the participants. Another camera (Nikon CoolPix $\mathrm{S} 203 ; 30 \mathrm{~Hz}$ ) was positioned in the frontal plane, $1 \mathrm{~m}$ away at $30 \mathrm{~cm}$-height, with a posterior view of the subject's feet. The third camera was the webcam of the computer where the EMG signal was being recorded (Sony Vaio PCG71911X). It was positioned two meters from the subjects in the sagittal plane at a $1 \mathrm{~m}$-height and primarily used to synchronize the EMG data with the kinematic measurements. Additional camera settings (e.g., shutter speed, zoom) were kept at their default values for all subjects and all trials. A light signal was used to synchronize the three cameras prior to each test. 


\section{Hip-abductors fatigue protocol}

After baseline squats were performed, participants were submitted to the hip-abductor fatigue protocol. They were positioned in the lateral decubitus position and performed four sets of side-lying hip abduction until concentric fatigue (10RM load), with a 2-min interval between sets. The cadence was controlled by the metronome, with each phase having the duration of two beats $(60 \mathrm{bpm})$. This exercise was chosen because it presents a high level of activation of the hip-abductor musculature [22]. Meanwhile, the protocol was chosen because it has been shown to be effective in decreasing strength in single-joint movements [23].

\section{Data analysis}

EMG data were analyzed using Miotec Suite (version 1.0, Miotec-Biomedical Equipment, Porto Alegre, Brazil) with a band-pass digital filter (5th order Butterworth with cut-off frequencies of 20-500 Hz) applied to the signals. The EMG signal was full-wave rectified and the Root Mean Square (RMS) of the activation during the MVICs was calculated, where the highest value was considered the maximal activation of each of the four muscles. For $\mathrm{SL}_{\mathrm{SQUAT}}$, the mean activation of three squats (2nd, 3rd and 4th) was used for evaluation, whereas the mean values of the three hops were evaluated in the $\mathrm{SL}_{\mathrm{HOP}}$ task. During both tasks, the RMS of the whole eccentric phases was calculated (using the synchronizing computer to define the beginning and end of the phase) and the mean activation value was computed for both the pre- and post-fatigue conditions.

All kinematic data were analyzed using Kinovea (version 0.8.15, Kinovea Organization, France). For both tasks, the difference between the angles measured in the final phase (end of the eccentric phase) and at the initial phase (unipedal support) was the variable of interest (joint displacement angle). The end of the eccentric phase was defined as the last frame before the subject starts to rise (initiating the concentric phase), identified in the sagittal view. The angle formed between the femur and the line that connects the two ASISs was adopted as the hip adduction angle, where greater negative values represented displacement towards adduction (Fig. 3A, B). The angle formed between the femur and the tibia was adopted as the knee FPPA, with greater negative values representing greater displacement towards knee valgus (Fig. 3A, B). The heel inversion displacement angle was calculated as the difference of the angle of tibiocalcaneal segments in bipedal support and the end of the eccentric phase of the single-leg tasks, where greater negative values represented greater displacement towards inversion (Fig. 3C, D).
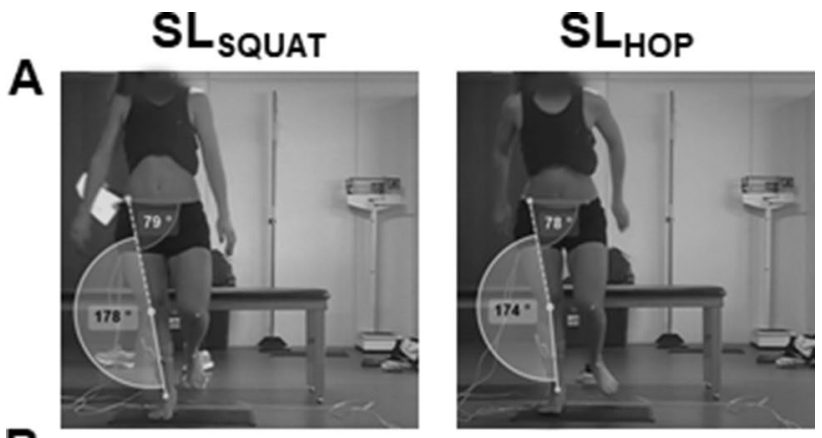

B
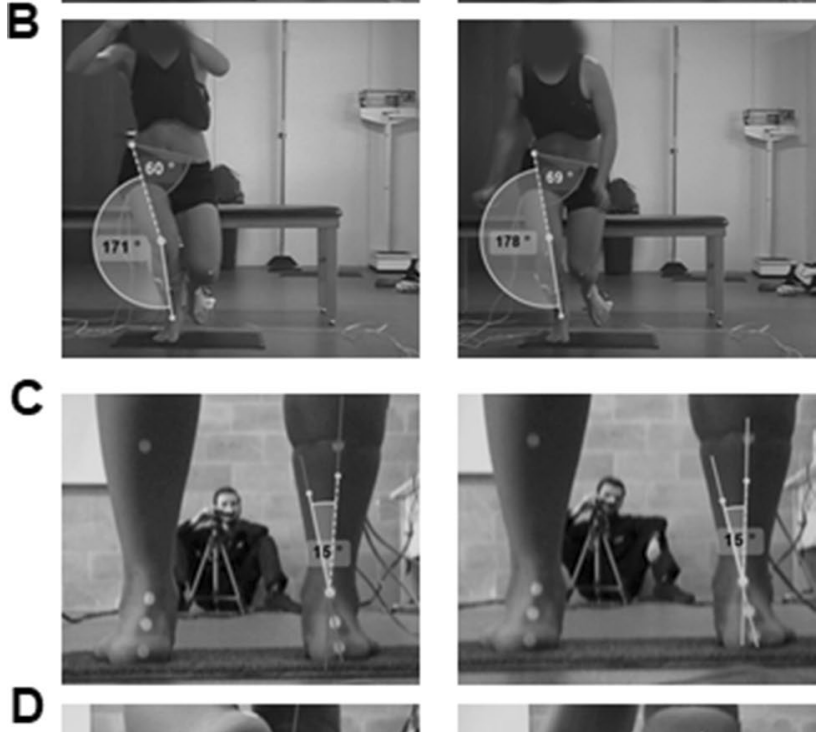

D
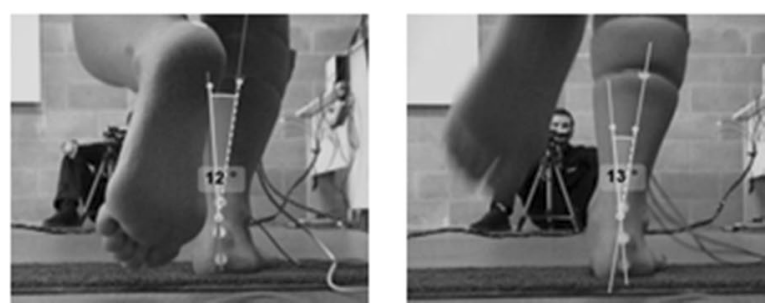

Fig. 3 Measurements of hip adduction and knee FPPA displacement angles (A and $\mathbf{B}$ ). Measurements of heel inversion displacement angle (C and D). The displacements were calculated by subtracting the values of the initial positions (A and $\mathbf{C}$ ) from final position values (B and D). Left panels show the $\mathrm{SL}_{\mathrm{SQUAT}}$ movements while right panels show the $\mathrm{SL}_{\mathrm{HOP}}$

\section{Statistical analysis}

A Shapiro-Wilk test was performed to verify the normality of the kinematic and electromyographical data. The sum of the activation of the two hip-abductor muscles (gluteus medius and tensor fascia latae) during the MVICs before and after the fatigue protocol was compared using a paired samples $t$-test [24]. Hedges “ $\mathrm{g}_{\mathrm{av}}$ ”, calculated from Cohen's " $\mathrm{d}_{\mathrm{av}}$ " was used to measure the effect sizes of these comparisons, values of $0.2,0.5$ and 0.8 were considered to be small, medium, and large, respectively [25]. A repeated measures two-way analysis of variance was used to evaluate differences between the two tasks at the pre- and post-fatigue 
moments (factors: task and moment) [26]. In addition, effect sizes (Partial Eta Squared " $\eta_{\mathrm{p}}{ }^{2}$ ") were calculated to assess the magnitude of the significant differences. Values $\geq 0.01$ indicate a small effect size, $0.06 \geq$ indicate medium effect size and $\geq 0.14$ indicate a large effect size [25]. Spearman's rank correlation coefficient was used to verify the association between the two tasks in the pre- and post-fatigue moments and were classified as follows: $<0.1=$ trivial, $0.1-0.3=$ small, $0.3-0.5=$ moderate and $>0.5=$ large. Analy ses were performed using SPSS 20.0 (SPSS Inc., Chicago, USA) while adopting a significance level of $0.05(5 \%)$.

\section{Results}

Seventeen participants (nine women) were recruited and completed all portions of the study (age: $28.41 \pm 6.05$ years; body mass: $71.91 \pm 12.88 \mathrm{~kg}$; height: $1.69 \pm 0.08 \mathrm{~m}$; body fat: $22.57 \pm 4.47 \%$; strength training experience: $6.11 \pm 4.24$ years).

All data used in this manuscript are available as a supplementary material (Supplementary material 2). In the MVICs performed before and after the fatigue protocol, hip abductors (gluteus medius + tensor fascia latae) activation decreased significantly (pre: $1120.8 \pm 530.3$; post: $919.8 \pm 592.1 \mathrm{mV} ; P=0.012 ; \mathrm{g}_{\mathrm{av}}=0.34$, medium).

Hip adduction displacement toward adduction (Fig. 4A) was greater in $\mathrm{SL}_{\mathrm{SQUAT}}$ than in $\mathrm{SL}_{\mathrm{HOP}}\left(P=0.005 ; \eta_{\mathrm{p}}{ }^{2}=0.39\right.$, large). No differences were found between the pre- and postfatigue moments $(P=0.145)$ and no interaction was found between the factors $(P=0.610)$. The two tasks were not significantly correlated between each other in neither the prenor the post-fatigue moments (Fig. 4D).

Knee FPPA displacement (Fig. 4B) was not significantly different between tasks $(P=0.380)$ or moments $(P=0.243)$ and no interaction was found between factors $(P=0.523)$. No significant correlations were found between the two tasks in the pre- and post-fatigue moments (Fig. 4E).

There were no significant differences in heel inversion displacement angle (Fig. 4C) between tasks $(P=0.617)$, moments $(P=0.201)$ or interaction between factors $(P=0.175)$. The two tasks were positively correlated in both the pre-fatigue $\left(r_{\mathrm{s}}=0.746\right.$, large; $\left.P=0.001\right)$ and the post-fatigue moments $\left(r_{\mathrm{s}}=0.524\right.$, large; $\left.P=0.031\right)$ (Fig. $\left.4 \mathrm{~F}\right)$.

Normalized activation of the hip-abductor muscles during the tasks were not different between the pre- and post-fatigue moments (gluteus medius: $P=0.794$; tensor fascia latae: $P=0.975)$ and no interactions were found between factors (gluteus medius: $P=0.907$; tensor fascia latae: $P=0.502$ ). However, the gluteus medius muscle (Fig. 5A) had greater activation during the $\mathrm{SL}_{\mathrm{SQUAT}}$ than during the $\mathrm{SL}_{\mathrm{HOP}}$ $\left(P=0.008 ; \eta_{\mathrm{p}}{ }^{2}=0.36\right.$, large $)$. Meanwhile, tensor fascia latae activation (Fig. 5B) was also not different between tasks $(P=0.705)$.

For peroneus longus and tibialis anterior, there were no significant differences between moments (peroneus longus: $P=0.561$; tibialis anterior: $P=0.861$ ) or interactions (peroneus longus: $P=0.312$; tibialis anterior: $P=0.136$ ). However, both muscles presented differences between the tasks. Peroneus longus activation (Fig. 5C) was higher during $\mathrm{SL}_{\mathrm{SQUAT}}$ than $\mathrm{SL}_{\mathrm{HOP}}\left(P=0.037 ; \eta_{\mathrm{p}}^{2}=0.24\right.$, large $)$. Whereas tibialis anterior activation (Fig. 5D) was greater during $\mathrm{SL}_{\mathrm{HOP}}$ than $\mathrm{SL}_{\mathrm{SQUAT}}\left(P=0.037 ; \eta_{\mathrm{p}}{ }^{2}=0.66\right.$, large $)$.

\section{Discussion and conclusions}

In this study, we found that healthy young individuals present a greater hip adduction displacement when performing a slower single-leg task than when performing a faster one. We also observed that the knee and heel frontal plane angles do not differ when performing the two tasks nor after fatigue of the hip-abductor muscles. Furthermore, we identified that hip and knee displacement angles are not correlated between the two tasks, but the heel ones are. And finally, we found that the gluteus medius and the peroneus longus muscles are more activated during $\mathrm{SL}_{\mathrm{SQUAT}}$ than $\mathrm{SL}_{\mathrm{HOP}}$, whereas the opposite behavior was found for the tibialis anterior.

Excessive hip adduction can contribute to increased patellofemoral joint pressure, due to the resulting smaller contact areas [27]. It is also accepted that hip position affects the forces experienced by the knee joint [28], consequently also influencing injury risk. In our study, hip joint displacement towards adduction did not seem to be influenced by hipabductors' fatigue level. These results go against those found in previous studies that have evaluated the landing phase of single-leg tasks using hip-fatigue protocols. Jacobs et al. [12] found that a similar population increased hip adduction displacement angle when landing after a fatigue protocol, while females evaluated by Geiser et al. [3] increased hip adduction during the weight acceptance phase of three different landing tasks. These contrasting results are likely due to the more stable tasks performed in our study, since landing tasks may not allow participants to adapt their muscle activation levels to stabilize the pelvis prior to the start of the eccentric contractions. In contrast, during our tasks, in which participants were already supporting their weight in a single-leg stance prior to the beginning of the movement, they were able to reach similar activation levels of the gluteus medius and the tensor fascia latae in the pre- and postfatigue moments. In addition, the fatigue-induced weakness in the study may have been different. In our study, participants reported concentric failure at the end of each hipabduction exercise set and the activation of the hip-abductor muscles during the MVICs immediately after the fatiguing 

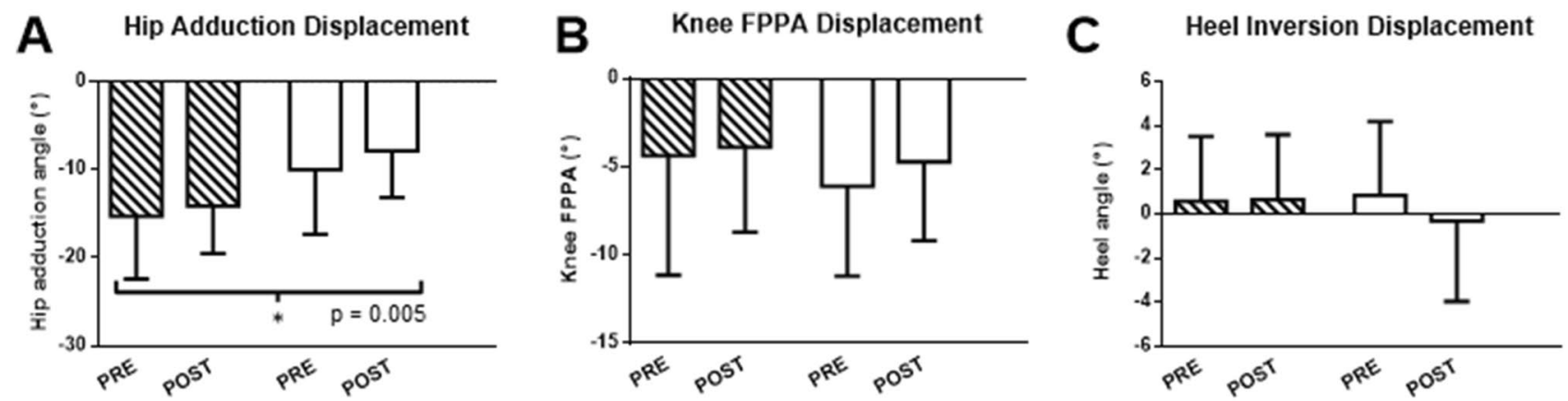

\section{$\varpi \nabla \mathrm{SL}_{\text {SQUAT }} \square \mathrm{SL}_{\text {HOP }}$}
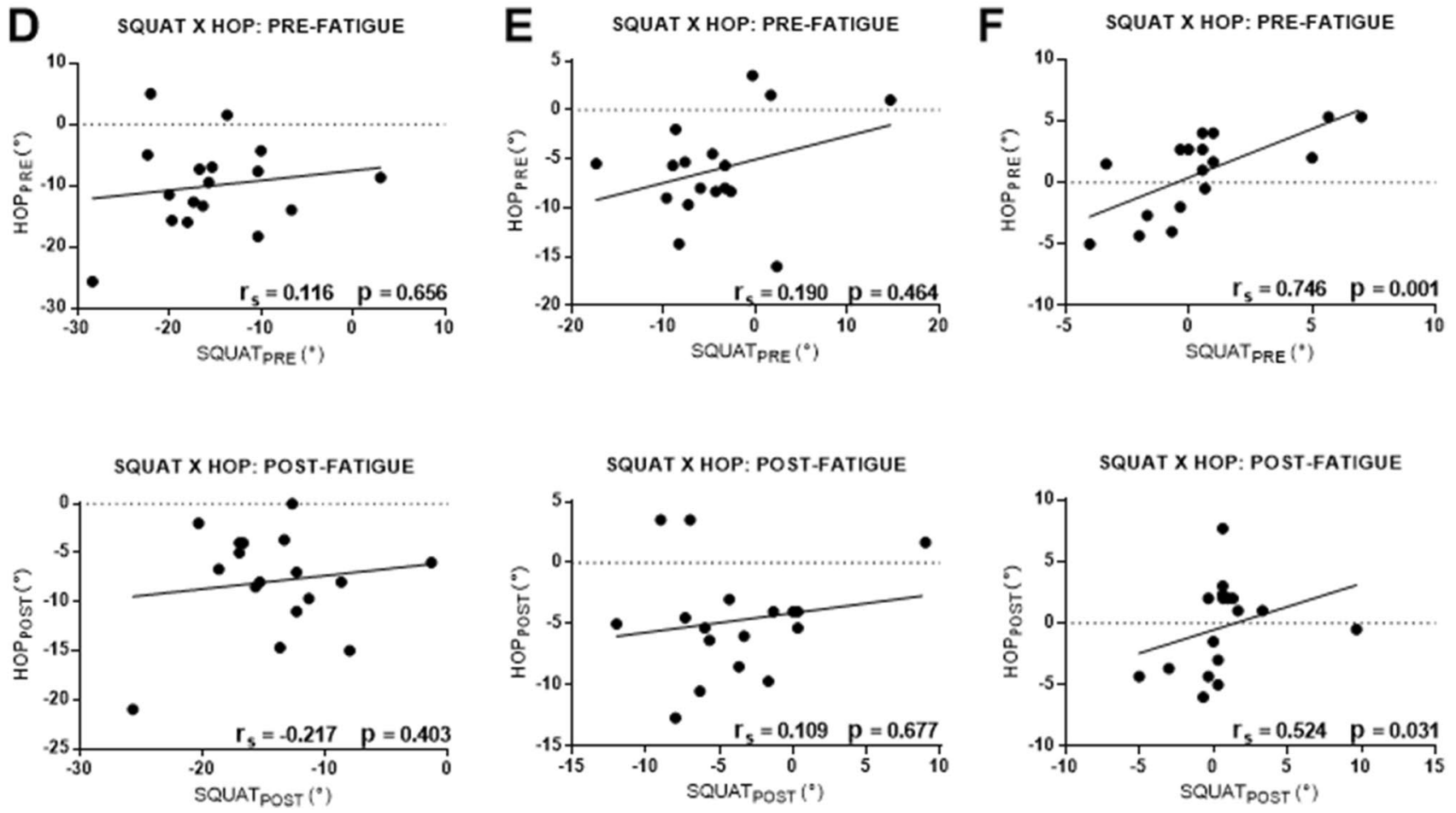

Fig. 4 Effects of tasks and moments on hip adduction angle (A), frontal plane projection angle $(\mathbf{B})$ and heel inversion angle $(\mathbf{C})$ displacement. Correlation between single-leg squat and single-leg hop meas-

protocol was decreased, indicating neuromuscular fatigue [24]. However, the percentage of fatigue-induced weakness at the moment of the exercises was not quantified. Therefore, there is a possibility that the participants in the other studies were less capable of producing hip-abduction force during their post-fatigue tasks [3, 12].

There were, however, differences between the two tasks on the hip joint. Hip adduction displacement was $5.7^{\circ}$ greater in the $\mathrm{SL}_{\mathrm{SQUAT}}$ than in the $\mathrm{SL}_{\mathrm{HOP}}$. Talarico et al. [29] found that both squat speed and squat depth influence center of pressure sway range and area in the mediolateral axis. Briefly, slower speeds and greater depths resulted in an increase in these dynamic postural control parameters [29]. urements in the pre- and post-fatigue moments at the hip (D), knee (E) and heel $(\mathbf{F}) .{ }^{*}=\mathrm{SL}_{\text {SQUAT }}$ higher than $\mathrm{SL}_{\mathrm{HOP}} \cdot n=17$

In our study, $\mathrm{SL}_{\mathrm{SQUAT}}$ had a controlled slower speed, and consequent greater duration, than the more dynamic $\mathrm{SL}_{\mathrm{HOP}}$. Furthermore, participants were instructed to squat as deep as possible during the $\mathrm{SL}_{\mathrm{SQUAT}}$, while no such instruction was given during the $\mathrm{SL}_{\mathrm{HOP}}$, since the goal was to jump forward. These characteristics probably required the participants to use greater hip adduction as a balance mechanism during the slower and deeper task $\left(\mathrm{SL}_{\mathrm{SQUAT}}\right)$, which is likely a consequence of the other segments (including the upper body) having more opportunity to shift throughout the movement, altering the position of the center of mass. Conversely, the $\mathrm{SL}_{\mathrm{HOP}}$, as it is a faster and less deep task, may have not been as influenced by other frontal plane moments, resulting in 
Fig. 5 Effects of tasks and moments on the activation of the gluteus medius (A), tensor fascia latae (B), peroneus longus $(\mathbf{C})$ and tibialis anterior $(\mathbf{D})$. * $=\mathrm{SL}_{\mathrm{SQUAT}}$ higher than $\mathrm{SL}_{\mathrm{HOP}}$; $\#=\mathrm{SL}_{\mathrm{HOP}}$ higher than $\mathrm{SL}_{\mathrm{SQUAT}}$. $n=17$
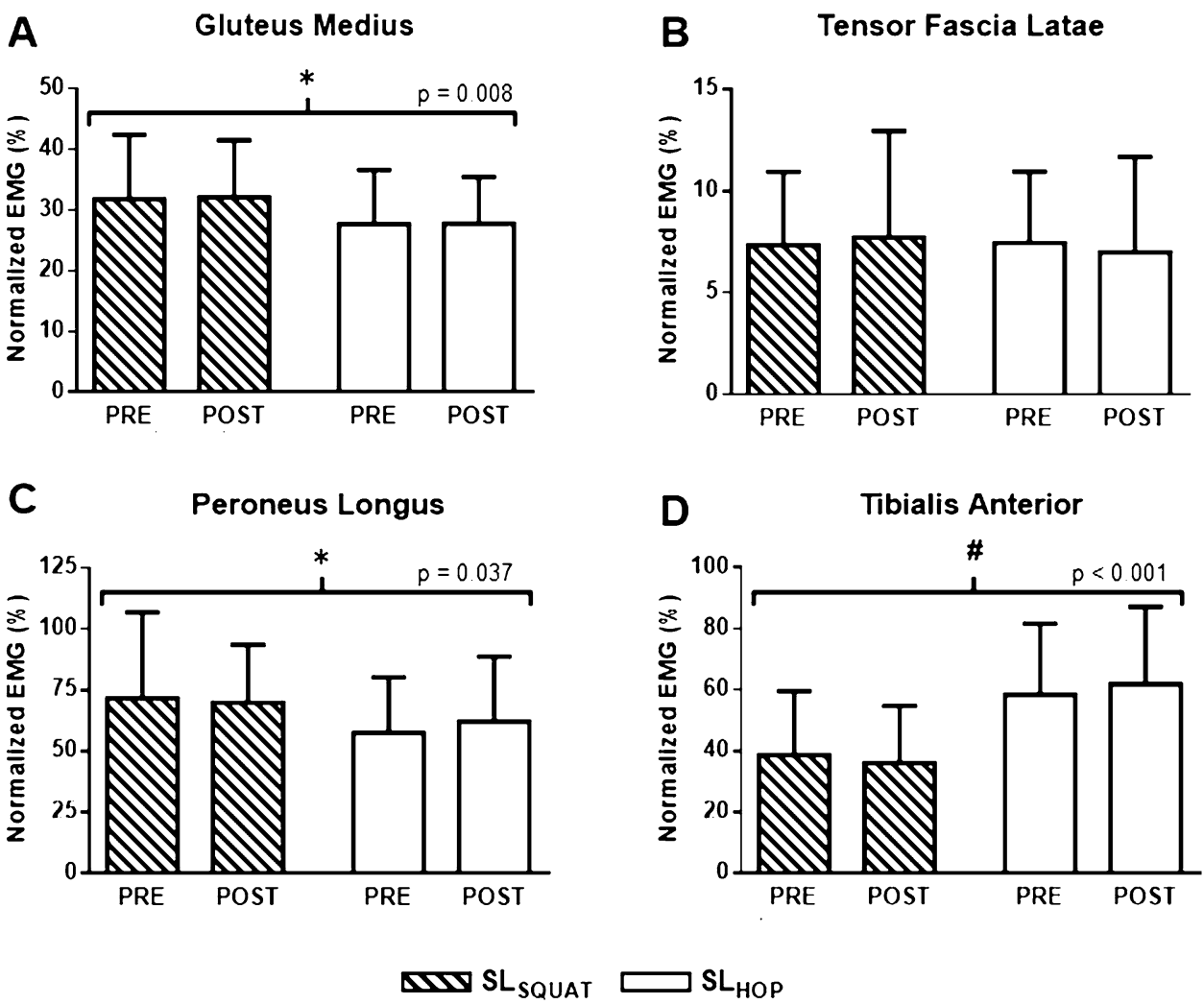

lower hip adduction displacement and lower activation of the gluteus medius muscle.

Excessive knee valgus has been associated with patellofemoral pain [1] and higher risk of anterior cruciate ligament injury in athletes [7]. In our study, the FPPA displacement did not change after fatigue of the hip-abductors. Our results agree with those of Jacobs et al. [12], who also did not find differences in knee abduction displacement. On the other hand, our results differ from those found by Geiser et al. [3] and by Patrek et al. [5], who, after hip-abductors fatigue, found greater abduction displacement during the weight acceptance phase and greater knee abduction angle $60 \mathrm{~ms}$ after initial contact, respectively. The tasks performed in these two studies involved a much higher vertical component than the ones performed by Jacobs et al. and by us. In the Geiser study, participants landed from a height equivalent to their maximal vertical countermovement jump, while in the Patrek study subjects consistently landed from a $40 \mathrm{~cm}$ height $[3,5]$. The landing performed by Jacobs was after a hop, with length and height of the jump being equivalent to $40 \%$ and $15 \%$ of their height, respectively (average height: $173.1 \mathrm{~cm}$ ) [12]. It is possible that, in tasks with a lesser vertical component, the adjustments required from the lower limbs to control the eccentric movement and maintain balance do not occur at the knee. In addition, the possible discrepancy in hip-abductor fatigue-induce weakness, in our study, may also have led to these differences.
Hip-abductor weakness has been associated with lateral ankle sprains (inversion sprains) [30], as increased postural sway has been regarded as an important risk factor for this type of injury [31]. In one of their studies, Lee and Powers [8] found increased center of pressure medial-lateral displacement in subjects with diminished hip-abductor strength during a balance task and, in their previous study, using a landing task, observed that the same was true when decreasing participants' strength using a hip-abductor fatigue protocol [13]. They have also reported an increased contribution of the peroneus longus muscle to compensate for the larger invertor and evertor moments that resulted from proximal instability [8], although invertor muscles were not measured. In our study, the displacement towards inversion was not significantly different after the fatigue protocol, suggesting that participants were able to maintain their ankle joints stable during the tasks.

When comparing the two tasks, there were also no differences in inversion displacement, however, significant differences in activation were observed. During the $\mathrm{SL}_{\mathrm{SQUAT}}$, peroneus longus activation was higher while tibialis anterior activation was lower than in $\mathrm{SL}_{\mathrm{HOP}}$. One possible explanation for these findings is that there could have been greater inversion moments during the slower task due to a higher balance requirement [29], and the higher peroneus longus activation and lower tibialis anterior activation could have been a strategy to maintain the joint in an optimal position. 
Conversely, during the faster task, there could have been eversion moments and the muscles could have behaved accordingly in order to stabilize the joint. However, this hypothesis should be further investigated in studies measuring ankle joint moments with the use of a force plate.

The displacement angles measured at the hip and the knee were not correlated between the two tasks. These results suggest that the two tasks performed at two speeds, even if they are somewhat similar, give different information regarding the frontal plane hip and knee angles. Some athletes may present greater lower limb misalignments when performing a more dynamic task with a forward goal while some might present greater values when performing a task that is slower. In practice, this means that athletes should be evaluated using tasks performed at different speeds, as using a single one might result in neglection of important information. The displacement angles measured at the heel were strongly correlated between the tasks. However, caution should be taken when extrapolating this result to clinical practice, since the values found during our two tasks were low and tasks with greater ankle inversion displacement may present lower correlation when performed at different speeds.

To the best of our knowledge, this was the first study that evaluated frontal plane lower limb alignment when executing two similar tasks performed at different speeds. Furthermore, we chose to use a fatigue protocol that has been shown to reduce force in single-joint muscles [23] and is within the recommendations for strength training from the American College of Sports Medicine [32]. However, we must acknowledge some limitations. Although two-dimensional analysis of lower limb kinematics in the frontal plane is valid and reliable [33], other measurements performed with threedimensional analysis have also been shown to be relevant to the risk of patellofemoral and ankle joint injuries [34]. Furthermore, the trunk position during the tasks influences the position of the CoM in relation to the base of support, and, consequently, the balance. Therefore, measurements of the upper body kinematics may have contributed to a better understanding of the CoM position and the demands that were being placed in the lower limbs throughout the movement. Furthermore, we were unable to measure the participants' level of force before and after the fatigue protocol, so we did not have a quantifiable measurement of how much weaker the muscles were after fatigue. However, the fatigue protocol consisted of four sets using a load that resulted in concentric failure at the tenth repetition and hip-abductor activation during post-fatigue MVIC was decreased, which indicates that a high level of neuromuscular fatigue was reached [24, 35-37]. Finally, even though localized muscle fatigue decreases muscle strength, it is not an exact replication of how individuals with hip-abductor weakness would perform during these tasks. Individuals with natural weakness would have had time to create compensatory mechanisms that our subjects did not. However, the fatigueinduced weakness generated in our protocol allowed an identification of a cause-and-effect relationship, which would not have been possible when evaluating participants that already presented hip-abductor weakness [5].

In the present study, we have found that participants presented greater hip adduction displacement when performing a slower task, likely because of a mechanism to maintain balance. We have also found that a hip-abductor fatigue protocol does not change lower limb alignment during a single-leg squat or during the propulsion phase of the single-leg hop. Finally, we found that hip and knee frontal plane displacement angles are not correlated between the two similar tasks performed at different speeds. Based on these results, clinicians should consider different options when measuring lower limb alignment. Since the hip and knee displacement angles are not correlated and significantly different proximal and distal muscle activation were found, it might be more appropriate to perform more than one task, so that information that could be found only in one of the speeds is not neglected.

Supplementary Information The online version contains supplementary material available at https://doi.org/10.1007/s11332-021-00854-4.

Author contributions RR wrote the original manuscript, performed data analysis, data interpretation, literature search and figure. $\mathrm{CN}$ and FS, IB, LF and JB performed literature search and contributed to the study design and data collection. RR performed literature search, designed the study, collected, analyzed, and interpreted the data and contributed to the writing of the original manuscript. All authors read and approved the final version of the manuscript.

Funding Open access funding provided by Università degli Studi di Milano within the CRUI-CARE Agreement. This study did not receive any specific funding from funding agencies in the public, commercial, or nonprofit sectors.

\section{Declarations}

Conflict of interest The authors have no conflicts of interest to declare that are relevant to the content of this article.

Ethical statement and Informed consent The study was approved by the University Research Ethics Board (Project number: 76759817.7.0000.5668) and was conducted in compliance with the standards set by the Declaration of Helsinki. All participants provided informed written consent prior to their participation in the study.

Open Access This article is licensed under a Creative Commons Attribution 4.0 International License, which permits use, sharing, adaptation, distribution and reproduction in any medium or format, as long as you give appropriate credit to the original author(s) and the source, provide a link to the Creative Commons licence, and indicate if changes were made. The images or other third party material in this article are included in the article's Creative Commons licence, unless indicated otherwise in a credit line to the material. If material is not included in the article's Creative Commons licence and your intended use is not 
permitted by statutory regulation or exceeds the permitted use, you will need to obtain permission directly from the copyright holder. To view a copy of this licence, visit http://creativecommons.org/licenses/by/4.0/.

\section{References}

1. Herrington L (2014) Knee valgus angle during single leg squat and landing in patellofemoral pain patients and controls. Knee 21:514-517. https://doi.org/10.1016/j.knee.2013.11.011

2. Alenezi F, Herrington L, Jones P, Jones R (2014) Relationships between lower limb biomechanics during single leg squat with running and cutting tasks: a preliminary investigation. Br J Sports Med 48:560-561. https://doi.org/10.1136/bjsports-2014-093494.3

3. Geiser CF, O'Connor KM, Earl JE (2010) Effects of isolated hip abductor fatigue on frontal plane knee mechanics. Med Sci Sports Exerc 42:535-545. https://doi.org/10.1249/MSS.0b013 e3181b7b227

4. Jacobs J, Jansen M, Janssen H, Raijmann W, Van Alfen N, Pillen S (2013) Quantitative muscle ultrasound and muscle force in healthy children: a 4-year follow-up study. Muscle Nerve 47:856-863. https://doi.org/10.1002/mus.23690

5. Patrek MF, Kernozek TW, Willson JD, Wright GA, Doberstein ST (2011) Hip-abductor fatigue and single-leg landing mechanics in women athletes. J Athl Train 46:31-42. https://doi.org/10. 4085/1062-6050-46.1.31

6. Almeida GPL, De Moura Campos Carvalho e Silva AP, França FJR, Magalhães MO, Burke TN, Marques AP (2016) Relationship between frontal plane projection angle of the knee and hip and trunk strength in women with and without patellofemoral pain. J Back Musculoskelet Rehabil 29:259-266. https://doi.org/ 10.3233/BMR-150622

7. Koga H, Nakamae A, Shima Y, Iwasa J, Myklebust G, Engebretsen L, Bahr R, Krosshaug T (2010) Mechanisms for noncontact anterior cruciate ligament injuries: knee joint kinematics in 10 injury situations from female team handball and basketball. Am J Sports Med 38:2218-2225. https://doi.org/10.1177/03635 46510373570

8. Lee SP, Powers CM (2014) Individuals with diminished hip abductor muscle strength exhibit altered ankle biomechanics and neuromuscular activation during unipedal balance tasks. Gait Posture 39:933-938. https://doi.org/10.1016/j.gaitpost. 2013.12.004

9. McLeish RD, Charnley J (1970) Abduction forces in the one-legged stance. J Biomech. https://doi.org/10.1016/00219290(70)90006-0

10. Neumann DA (2010) Kinesiology of the hip: a focus on muscular actions. J Orthop Sports Phys Ther 40:82-94

11. Runge CF, Shupert CL, Horak FB, Zajac FE (1999) Ankle and hip postural strategies defined by joint torques. Gait Posture 10:161-170. https://doi.org/10.1016/S0966-6362(99)00032-6

12. Jacobs CA, Uhl TL, Mattacola CG, Shapiro R, Rayens WS (2007) Hip abductor function and lower extremity landing kinematics: sex differences. J Athl Train 42:76-83

13. Lee SP, Powers C (2013) Fatigue of the hip abductors results in increased medial-lateral center of pressure excursion and altered peroneus longus activation during a unipedal landing task. Clin Biomech 28:524-529. https://doi.org/10.1016/j.clinbiomech. 2013.04.002

14. Ha PL, Dalton BE, Alesi MG, Smith TM, VanDusseldorp TA, Feito Y, Hester GM (2021) Isometric versus isotonic contractions: sex differences in the fatigability and recovery of isometric strength and high-velocity contractile parameters. Physiol Rep 9:e14821. https://doi.org/10.14814/phy2.14821
15. Akagi R, Hinks A, Davidson B, Power GA (2020) Differential contributions of fatigue-induced strength loss and slowing of angular velocity to power loss following repeated maximal shortening contractions. Physiol Rep 8:e14362. https://doi.org/ $10.14814 /$ phy 2.14362

16. Augustsson J, Thomeé R, Lindén C, Folkesson M, Tranberg R, Karlsson J (2006) Single-leg hop testing following fatiguing exercise: reliability and biomechanical analysis. Scand J Med Sci Sport 16:111-120. https://doi.org/10.1111/j.1600-0838. 2005.00446.x

17. Bley AS, Correa JCF, Dos RAC, Rabelo NDDA, Marchetti PH, Lucareli PRG (2014) Propulsion Phase of the single leg triple hop test in women with patellofemoral pain syndrome: a biomechanical study. PLoS ONE 9:e97606. https://doi.org/10.1371/ journal.pone.0097606

18. Frigotto M, Cardoso C, Santos R, Rodrigues R (2019) Gluteus medius and tensor fascia latae muscle activation levels during multi-joint strengthening exercises. Mot Rev Educ Física 25:1-7

19. Hermens HJ, Freriks B, Disselhorst-Klug C, Rau G (2000) Development of recommendations for SEMG sensors and sensor placement procedures. J Electromyogr Kinesiol 10:361-374. https:// doi.org/10.1016/S1050-6411(00)00027-4

20. Fitarelli L, Ramos G, Scudiero F, Rabello R, Rodrigues R (2020) Core muscles thickness is not associated with knee frontal plane projection angle during single-leg squat in healthy people. Gait Posture 80:292-297. https://doi.org/10.1016/j.gaitpost.2020.06. 015

21. Perry SD, Lafortune MA (1995) Influences of inversion/eversion of the foot upon impact loading during locomotion. Clin Biomech 10:253-257. https://doi.org/10.1016/0268-0033(95)00006-7

22. Selkowitz DM, Beneck GJ, Powers CM (2013) Which exercises target the gluteal muscles while minimizing activation of the tensor fascia lata? Electromyographic assessment using fine-wire electrodes. J Orthop Sports Phys Ther 43:54-64. https://doi.org/ 10.2519/jospt.2013.4116

23. Baroni BM, Pompermayer MG, Cini A, Peruzzolo AS, Radaelli R, Brusco CM, Pinto RS (2017) Full range of motion induces greater muscle damage than partial range of motion in elbow flexion exercise with free weights. J Strength Cond Res 31:2223-2230. https:// doi.org/10.1519/JSC.0000000000001562

24. Rodrigues R, de Azevedo FR, Teixeira BC, Cauduro Oliveira Macedo R, Diefenthaeler F, Baroni BM, Vaz MA (2019) Can the combination of acute alcohol intake and one night of sleep deprivation affect neuromuscular performance in healthy male adults? A cross-over randomized controlled trial. J strength Cond Res 33:1244-1251. https://doi.org/10.1519/JSC.0000000000003124

25. Pautz N, Olivier B, Steyn F (2018) The use of parametric effect sizes in single study musculoskeletal physiotherapy research: a practical primer. Phys Ther Sport 32:87-97. https://doi.org/10. 1016/j.ptsp.2018.05.002

26. Cohen J (1988) Statistical power analysis for the behavioral sciences, 2nd ed. Lawrence Earlbaum Associates, Hillsdale

27. Powers CM (2003) The influence of altered lower-extremity kinematics on patellofemoral joint dysfunction: a theoretical perspective. J Orthop Sports Phys Ther 33:639-646. https://doi.org/10. 2519/jospt.2003.33.11.639

28. Griffin LY, Agel J, Albohm MJ, Arendt EA, Dick RW, Garrett WE, Garrick JG, Hewett TE, Huston L, Ireland ML, Johnson RJ, Kibler WB, Lephart S, Lewis JL, Lindenfeld TN, Mandelbaum BR, Marchak P, Teitz CC, Wojtys EM (2000) Noncontact anterior cruciate ligament injuries: risk factors and prevention strategies. $\mathrm{J}$ Am Acad Orthop Surg 8:141-150. https://doi.org/10.5435/00124 635-200005000-00001

29. Talarico MK, Lynall RC, Mauntel TC, Wasserman EB, Padua DA, Mihalik JP (2019) Effect of single-leg squat speed and depth on 
dynamic postural control under single-task and dual-task paradigms. J Appl Biomech 35:272-279. https://doi.org/10.1123/jab. 2018-0327

30. Friel K, McLean N, Myers C, Caceres M (2006) Ipsilateral hip abductor weakness after inversion ankle sprain. J Athl Train 41:74-78

31. Kristianslund E, Bahr R, Krosshaug T (2011) Kinematics and kinetics of an accidental lateral ankle sprain. J Biomech 44:25762578. https://doi.org/10.1016/j.jbiomech.2011.07.014

32. Garber CE, Blissmer B, Deschenes MR, Franklin BA, Lamonte MJ, Lee IM, Nieman DC, Swain DP (2011) Quantity and quality of exercise for developing and maintaining cardiorespiratory, musculoskeletal, and neuromotor fitness in apparently healthy adults: guidance for prescribing exercise. Med Sci Sports Exerc 43:1334-1359. https://doi.org/10.1249/MSS.0b013e318213fefb

33. Munro A, Herrington L, Carolan M (2012) Reliability of 2-dimensional video assessment of frontal-plane dynamic knee valgus during common athletic screening tasks. J Sport Rehabil 21:7-11. https://doi.org/10.1123/jsr.21.1.7

34. Aerts I, Cumps E, Verhagen E, Verschueren J, Meeusen R (2013) A systematic review of different jump-landing variables in relation to injuries. J Sports Med Phys Fitness 53:509-519
35. Rodrigues R, Baroni BM, Pompermayer MG, de Oliveira LR, Geremia JM, Meyer F, Vaz MA (2014) Effects of acute dehydration on neuromuscular responses of exercised and nonexercised muscles after exercise in the heat. J strength Cond Res 28:3531-3536. https://doi.org/10.1519/JSC.0000000000000578

36. Rossato M, Dellagrana RA, Sakugawa RL, Lazzari CD, Baroni BM, Diefenthaeler F (2018) Time response of photobiomodulation therapy on muscular fatigue in humans. J strength Cond Res 32:3285-3293. https://doi.org/10.1519/JSC.0000000000002339

37. Girard O, Racinais S (2014) Combining heat stress and moderate hypoxia reduces cycling time to exhaustion without modifying neuromuscular fatigue characteristics. Eur J Appl Physiol 114:1521-1532. https://doi.org/10.1007/s00421-014-2883-0

Publisher's Note Springer Nature remains neutral with regard to jurisdictional claims in published maps and institutional affiliations. 